\title{
SKY-DISTRIBUTION OF INTENSITY OF SYNCHROTRON RADIO EMISSION OF RELATIVISTIC ELECTRONS TRAPPED IN EARTH'S MAGNETIC FIELD
}

\author{
V.V. Klimenko \\ Institute of Applied Physics RAS \\ Nyzhny Novgorod, Russia, klimenko@appl.sci-nnov.ru
}

\begin{abstract}
This paper presents the calculations of synchrotron radio emission intensity from Van Allen belts with Gaussian space distribution of electron density across L-shells of a dipole magnetic field, and with Maxwell's relativistic electron energy distribution. The results of these calculations come to a good agreement with measurements of the synchrotron emission intensity of the artificial radiation belt's electrons during the Starfish nuclear test. We have obtained two-dimensional distributions of radio brightness in azimuth — zenith angle coordinates for an observer on Earth's surface. The westside and eastside intensity maxima exceed several times the maximum level of emission in the meridian plane. We have also constructed two-dimensional
\end{abstract}

distributions of the radio emission intensity in decibels related to the background galactic radio noise level. Isotropic fluxes of relativistic electrons $(E \sim 1 \mathrm{MeV})$ should be more than $10^{7} \mathrm{~cm}^{-2} \mathrm{~s}^{-1}$ for the synchrotron emission intensity in the meridian plane to exceed the cosmic noise level by $0.1 \mathrm{~dB}$ (riometer sensitivity threshold).

Keywords: synchrotron radio emission, relativistic electrons, radiation belt, dipole magnetic field.

\section{INTRODUCTION}

The discovery of Earth's radiation belts in 1958 stimulated a series of studies aimed at calculating characteristics of synchrotron radio emission of electrons trapped in a dipole magnetic field [Dyce, Nakada, 1959; Korchak, 1963; Vesecky, Peterson, 1967]. In 1962, a high-altitude nuclear explosion in near-Earth space carried out during the Starfish nuclear test produced an artificial radiation belt (RB) consisting of high-energy electrons [Van Allen et al., 1963 ; Hess, 1963]. Synchrotron radio emission of these electrons [Ochs et al., 1963; Dyce, Horowitz, 1963; Peterson, Hower, 1963] was detected with special receivers - riometers [Little, Leinbach, 1958; Driatskii 1974], located at equatorial stations.

Because of the complexity of the calculations, characteristics of the RB synchrotron emission were computed for simple special cases: a) emission detection with an antenna located at the equator and directed to the zenith [Dyce, Nakada, 1959; Peterson, Hower, 1963]; b) detection of emission from outside the magnetosphere from large distances, i.e. as from a radioastronomical object [Korchak 1963; Vesecky, Peterson, 1967]. These calculations showed that in ordinary conditions of the terrestrial RB, its synchrotron emission intensity is substantially lower than the background level of cosmic noise. At the same time, during strong magnetospheric disturbances, subauroral stations sometimes recorded a rather intense natural ionosphericmagnetospheric radio noise in a frequency range 20$200 \mathrm{MHz}$ [Chivers, Wells, 1959; Egan, Peterson, 1960 ; Ellyett, 1969 ; Eriksen, Harang, 1969] morphologically similar to the synchrotron emission from the artificial
RB. Presumably, in special extreme situations such as a nuclear explosion in the magnetosphere and during strong magnetospheric disturbances, the synchrotron emission of relativistic magnetospheric electrons can make a noticeable addition to the cosmic background level and be recorded with ground-based receivers over a wide frequency range.

The practical possibility of detecting synchrotron emission from electrons of the natural RB is of interest from the point of view of diagnostics of the magnetosphere and study of electron acceleration in near-Earth space. Also of particular interest is the possibility of monitoring extreme conditions in satellite orbits because it is electrons of relativistic energies that create the main radiation background for on-board equipment ("killer electrons”) [Romanova et al., 2005; Potapov, 2017]. The purpose of this paper is to quantify the intensity of synchrotron emission from RB with a dipole magnetic field and Maxwell's relativistic electron energy distribution, as well as to obtain a picture of spatial skydistribution of emission intensity for a terrestrial observer, depending on the latitude of an observation station and on RB configuration.

\section{SYNCHROTRON RADIO EMISSION} OF THE ARTIFICIAL RADIATION BELT AND NATURAL HF/VHF RADIO EMISSION OF THE DISTURBED MAGNETOSPHERE

On July 9, 1962 at 09:00:09 UT at an altitude of 400 $\mathrm{km}$ over Johnston island in the Pacific Ocean (USA, $\left.\varphi \approx 16.7^{\circ} \mathrm{N}, \lambda \approx 169.5^{\circ} \mathrm{W}\right)$, an experimental nuclear explosion (1.4 Mt TNT equivalent) was carried out. It produced an artificial RB with maximum intensity 1.2-1.6 in $L$-shells ( $L$ is McIlwain's parameter) [Van Allen et al., 
1963; Hess, 1963]. Figure 1 shows a record of the synchrotron radio emission of a cloud of relativistic electrons formed during the nuclear explosion [Ochs et al., 1963]. The receiver frequency is $50 \mathrm{MHz}$ (riometer with $144 \mathrm{~m} \times 288 \mathrm{~m}$ antenna array); the recording point is the Radio Astronomy Observatory in Jicamarca (Peru, $\left.\varphi \approx 11.95^{\circ} \mathrm{S}, \lambda \approx 76.87^{\circ} \mathrm{W}\right)$ located at the geomagnetic equator (geomagnetic latitude $\Phi_{0} \approx 0.8^{\circ}$ ) and by $\Delta \lambda \approx 93^{\circ}$ in longitude to the east of the explosion site. The first maximum of the radio emission intensity, recorded approximately $6 \mathrm{~min}$ after the explosion, corresponds to the first arrival of the electron cloud caused by the east (gradient) drift from the explosion site. The second, less intense, maximum $30 \mathrm{~min}$ after the explosion was caused by electrons that had made a complete revolution around Earth. In 1-2 hrs after the explosion, the electron cloud was evenly distributed along L-shells around Earth. Then, the emission level gradually decreased in proportion to the time factor $(1+t / \tau)^{-1}$ with a characteristic time $\tau \approx 60$ days [Ochs et al., 1963].

The noise temperature of a riometer antenna is calculated by the formula [Driatskii, 1974] $k_{\mathrm{B}} T_{\mathrm{A}}=e I_{\mathrm{d}} R_{\mathrm{d}} / 2\left(k_{\mathrm{B}}\right.$ is the Boltzmann constant, $e$ is the electron charge, $R_{\mathrm{d}}$ is the noise diode load resistance, $I_{\mathrm{d}}$ is the noise diode current). Given the antenna efficiency $\eta_{\mathrm{a}}=0.63$ and $R_{\mathrm{d}}=52$ Ohm [Ochs et al., 1963], the effective temperature of the noise recorded in Jicamarca

$$
T_{\text {eff }}(\mathrm{K})=e R_{\mathrm{d}} I_{\mathrm{d}} /\left(2 k_{\mathrm{B}} \eta_{\mathrm{a}}\right) \approx 4.8 \cdot 10^{2} I_{\mathrm{d}}(\mathrm{MA}) .
$$

Then the background cosmic noise temperature before the explosion (see Figure 1) $T_{\text {eff }} \approx 5700 \mathrm{~K}\left(I_{\mathrm{d}} \approx 12\right.$ $\mathrm{mA})$. The excess of the noise temperature over the background at the first maximum corresponds to $\Delta T_{\text {eff }}$ $\approx 4.45 \cdot 10^{4} \mathrm{~K}\left(I_{\mathrm{d}} \approx 105 \mathrm{~mA}\right)$; at the second maximum, to $\Delta T_{\text {eff }} \approx 1.1 \cdot 10^{4} \mathrm{~K}\left(I_{\mathrm{d}} \approx 35 \mathrm{~mA}\right)$; and 3 hours after the explosion, to $\Delta T_{\text {eff }} \approx 8.6 \cdot 10^{3} \mathrm{~K}\left(I_{\mathrm{d}} \approx 30 \mathrm{~mA}\right)$.
Examples of characteristics of the natural radio emission driven by magnetospheric disturbances are given in [Chivers, Wells, 1959; Egan, Peterson, 1960; Ellyett, 1969; Eriksen, Harang, 1969]. In [Egan, Peterson, 1960], three receivers (27 MHz riometers), located approximately on one meridian at geomagnetic latitudes $\Phi_{0} \approx 62^{\circ}, 53^{\circ}$, and $43^{\circ}$ (Canada, USA), during strong magnetospheric disturbances $\left(K_{\mathrm{p}}=8^{+}\right)$simultaneously recorded absorption of cosmic radio emission at a latitude of $62^{\circ}$, caused by energetic electron precipitation $(E>40 \mathrm{keV})$, and bursts of noise emission correlated with the absorption at a latitude of $43^{\circ}$. The characteristic emission intensity was about $1-2 \mathrm{~dB}$ relative to the cosmic noise level. At an intermediate station at a latitude of $53^{\circ}$, either the absorption or the emission was observed in different intervals. Figure 2 shows the ionospheric-magnetospheric radio noise recoded at the Christchurch Observatory (New Zealand, $\Phi_{0} \approx 51^{\circ} \mathrm{S}$ ) [Ellyett, 1969 ]. Two antennas, one of which was directed to the zenith and the second to the south magnetic pole at an elevation angle of $11^{\circ}$, were alternately connected to the receiver $(43 \mathrm{MHz})$ at an interval of $4 \mathrm{~min}$. Radio noise from a high latitude region occurred simultaneously with the substorm in auroras and lasted for several hours. In the zenith direction there were no ionospheric disturbances and emission at that time. Then, with an increase in magnetospheric disturbance level and expansion of the zone of precipitation of energetic magnetospheric electrons in the equatorial direction, disturbances in the upper atmosphere at $06 \mathrm{LT}$ reached the Christchurch latitude. At that time, cosmic noise absorption was recorded in both directions (southern and zenith). It was induced by precipitation of electrons with energies $E>40 \mathrm{keV}$ from the magnetosphere to the upper atmosphere.

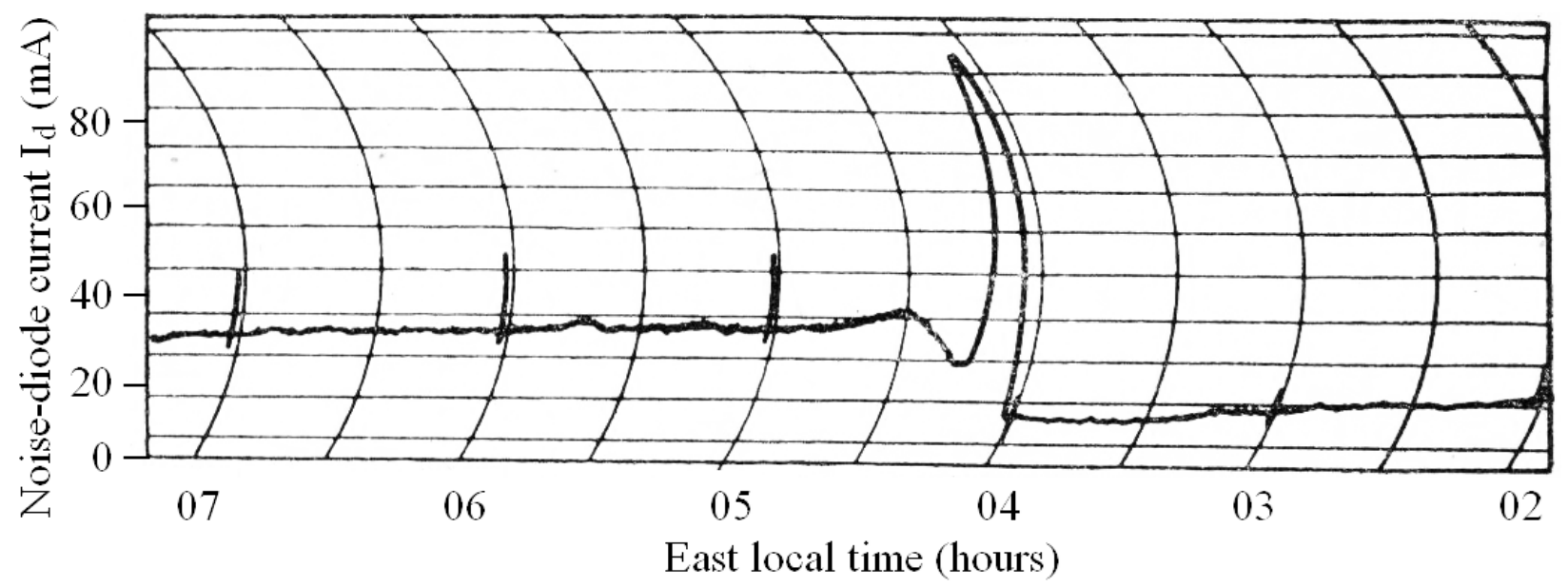

Figure 1. Synchrotron radio emission of the artificial RB at a frequency of $50 \mathrm{MHz}$ in Jicamarca (Peru) on July 9, 1962 [Ochs et al., 1963] (right-to-left time reading). 

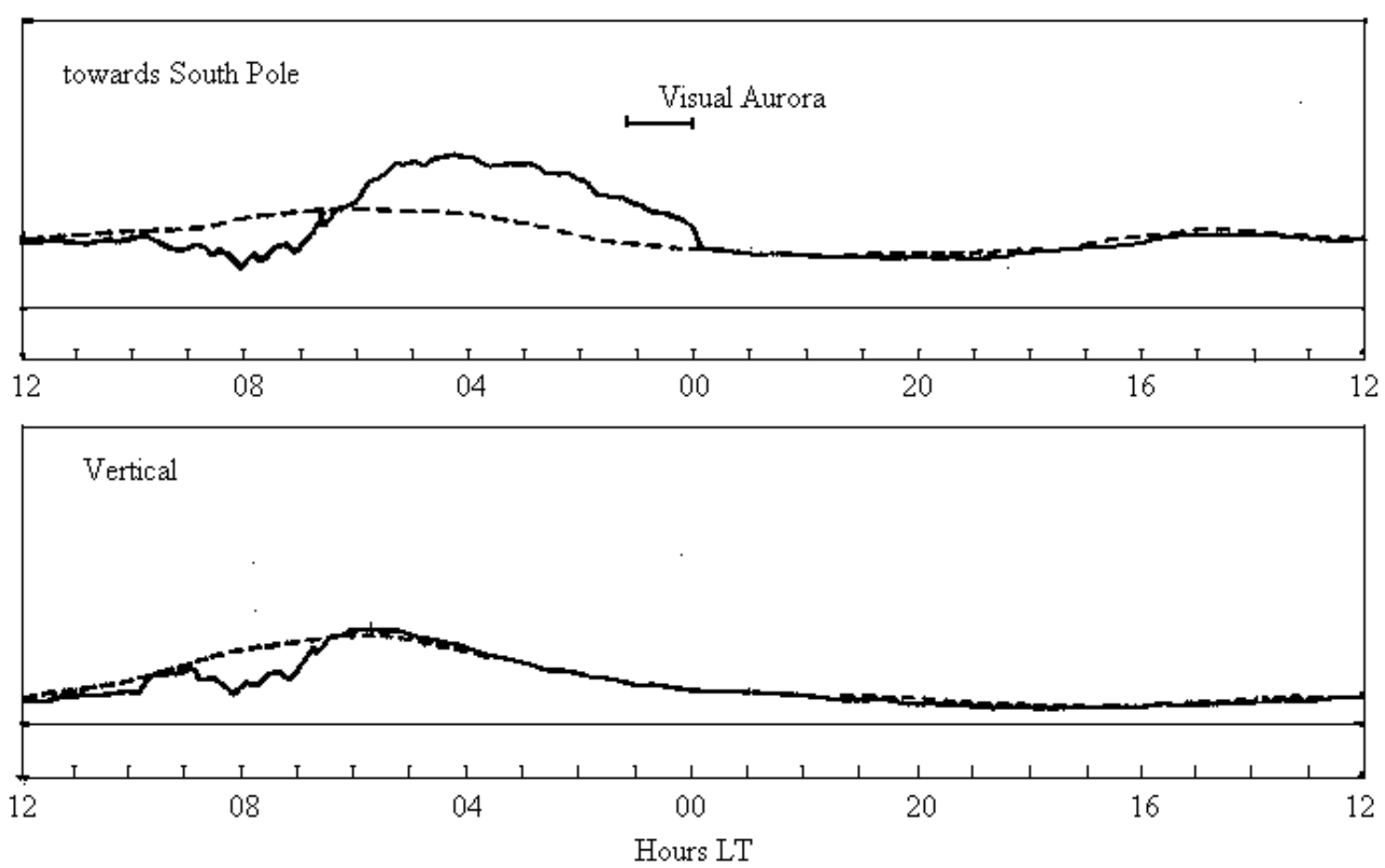

Figure 2. Radio noise recorded in Christchurch (New Zealand) on March 10, 1961 at a frequency of $43 \mathrm{MHz}$ [Ellyett, 1969 ] with an antenna directed to the south (top) and to the zenith. The dashed curve is the background level of galactic noise (right-toleft time reading)

Referring to Figure 2, the intensity of the radio noise from the high-latitude ionosphere was 20-30 \% (about 1 $\mathrm{dB}$ ) from the cosmic emission level or $\Delta T_{\text {eff }} \sim 2000-3000$ $\mathrm{K}$ at the background cosmic noise temperature $T_{\mathrm{N}} \approx 10000 \mathrm{~K}$ (at $43 \mathrm{MHz}$ ).

In [Chivers, Wells, 1959], the radio emission was recorded at the Jodrell Bank Observatory $\left(\mathrm{UK}, \Phi_{0} \approx 51^{\circ}\right)$ at a frequency of $80 \mathrm{MHz}$ with four antennas from four different directions: from the zenith, $\pm 60^{\circ}$ from the zenith in the plane of the geomagnetic meridian, and in the direction of the powerful radio source in Cassiopeia. During very strong magnetospheric disturbances, emission (about 1-2.5 dB above the cosmic noise level) from one direction and cosmic noise absorption from the other were simultaneously recorded. Eriksen, Harang [1969] described a case of radio noise recorded at 225 $\mathrm{MHz}$ (about 1.5-2 dB above the cosmic noise level) from the auroral ionosphere during a strong magnetospheric substorm at the end of a polar cap absorption event.

The cumulative evidence allows us to draw the following conclusions: 1) the natural magnetospheric HF radio emission occurs during very strong disturbances and concurs with precipitation of energetic electrons from the magnetosphere; 2) the radio emission intensity is non-uniformly distributed over the sky; 3) the frequency dependence of magnetospheric radio emission intensity in the range $27-225 \mathrm{MHz}$ is similar to the galactic noise spectrum because it is $\sim 1-2 \mathrm{~dB}$ above its level at all usable frequencies. It is known that in the frequency range 20-200 $\mathrm{MHz}$ the background cosmic radio noise is generated by synchrotron emission of relativistic electrons in galactic magnetic fields. Strictly speaking, there is no direct evidence that the natural magnetospheric HF radio emission is precisely the synchrotron emission of magnetospheric relativistic elec- trons. However, taking into account the similarity of the frequency dependence of the ionospheric-magnetospheric radio noise to the spectrum of synchrotron galactic radio emission and the morphological similarity to the synchrotron emission of the artificial RB (Figures 1 and 2), we can assume that the described cases of natural ionospheric-magnetospheric HF radio emission are most likely to be the synchrotron radio emission of relativistic electrons whose fluxes in the magnetosphere increase substantially during strong disturbances.

\section{CALCULATION OF INTENSITY OF SYNCHROTRON EMISSION FROM RELATIVISTIC ELECTRONS IN THE GEOMAGNETIC FIELD}

Ochs et al. [1963] have shown that in the decameter range (30-50 MHz) a low degree of polarization of the synchrotron emission from the artificial RB could be observed only at the equator in the near-zenith region, where the line of sight was orthogonal to the magnetic field and conditions of quasi-transverse (relative to the magnetic field) propagation held for radio waves. Even at small deviations from the orthogonality (more than $\pm 0.2^{\circ}$ ), quasi-transverse propagation conditions were violated and for a terrestrial observer the polarization became fairly chaotic due to the Faraday effect. Therefore, in practice with good approximation it suffices to calculate the total intensity of synchrotron emission without separation into polarized components. To calculate synchrotron emission characteristics, we use known expressions for spectral intensity $I_{v}\left(\mathrm{erg} \cdot \mathrm{cm}^{-2} \cdot \mathrm{sr}^{-1}\right)$ and brightness temperature $T_{\text {eff }}(\mathrm{K})$ [Ginsburg, Syrovatsky, 1965; Pakholchik, 1973]: 


$$
\begin{gathered}
I_{v}=\frac{\sqrt{3} e^{3}}{m c^{2}} \int d r H \sin \mu \int N(r, \varepsilon, \alpha) F\left(\frac{v}{v_{\mathrm{c}}}\right) d \varepsilon, \\
k_{\mathrm{B}} T_{\text {eff }}=\frac{c^{2}}{2 v^{2}} I_{v},
\end{gathered}
$$

where $e, m$ are the electron charge and mass, $c$ is the velocity of light, $r$ is the coordinate along the line of sight with the origin at abserving station, $N(\vec{R}, \varepsilon, \alpha)$ is the density of electrons in configuration space of energy, pitch angles and coordinates in the system with the origin at the center of Earth; the coordinates $r$ and $\vec{R}$ are connected by transformation formulas for transitions between the coordinate systems adopted (please find below),

$\varepsilon=m c^{2} / \sqrt{1-v^{2} / c^{2}}=\gamma m c^{2}$ is the total electron energy, $F(x)=x \int_{x}^{\infty} K_{5 / 3}(\xi) d \xi$ is the universal function of the synchrotron radiation theory, often used in tabulated form [Pakholchik, 1973], $K_{5 / 3}(x)$ is the McDonald function of the order of $5 / 3 ; H=H(r)$ is the value of the magnetic field at a radiation source point; $\mu=\mu(r)$ is the angle between magnetic field and emission directions, $v$ is the frequency, $v_{\mathrm{c}}=v_{\mathrm{c}}(r, \varepsilon)$ is the characteristic frequency:

$$
v_{\mathrm{c}}=\frac{3}{4 \pi} \frac{e H \sin \mu}{m c}\left(\frac{\varepsilon}{m c^{2}}\right)^{2} \text {. }
$$

In astrophysical applications, the energy distribution of electrons is assumed to be power-series, and it is taken that $\varepsilon>>m c^{2}(\gamma>>1)$. Therefore, without great error integral (1) is computed within $0 \leq \varepsilon \leq \infty$ in closed form [Ginzburg, Syrovatsky, 1965; Pakholchik, 1973]. In Earth's radiation belts, the exponential distribution $f$ $(E) \sim \exp \left(-E / E_{0}\right)$ is valid for energetic electrons, where $E_{0}$ is the kinetic energy measured by detectors, which is of the order of several tens or hundreds of kiloelectronvolts, whereas the total energy of electrons $\varepsilon$ slightly exceeds their rest energy. Therefore, energy integration in (1) should be performed from $m c^{2}$ to $\infty(1 \leq \gamma \leq \infty)$. An approximation for the exponential distribution is Maxwell's distribution whose relativistic form is

$$
N(\gamma, \vec{R}, \alpha) d \gamma d \Omega=\frac{N(\vec{R}, \alpha)}{\tau K_{2}(1 / \tau)} e^{-\frac{\gamma}{\tau}} \gamma \sqrt{\gamma^{2}-1} d \gamma d \Omega,
$$

where $\gamma=\varepsilon /\left(m c^{2}\right) \tau=k_{\mathrm{B}} T_{\mathrm{e}} /\left(m c^{2}\right), T_{\mathrm{e}}$ is the electron temperature, $K_{2}(x)$ is the second-order McDonald function, $d \Omega$ is the element of solid angle. Calculations of intensity (1) with distribution (2) in the ultrarelativistic approximation $(\gamma>>1)$ are presented in [Pakholchik, 1973]; a weakly relativistic case $\left(\gamma-1<1, \gamma^{3}<<q, q\right.$ is the harmonic number in the spectrum) under the simplifying condition $\mu=\pi / 2$ was examined in [Trubnikov, 1958].

We believe as in [Korchak, 1963 ] that the relativistic electron density in the magnetosphere has a Gaussian distribution in the equatorial plane in the radial direction and a pitch angle distribution $\sim \sin ^{n} \alpha$. Then, the change in the electron density in a magnetic field tube with $L$ will take the form

$$
N(\vec{R}, \alpha)=N_{\mathrm{m}} \exp \left[-\left(\frac{L-L_{m}}{\Delta L}\right)^{2}\right] h_{e}^{-\frac{n}{2}} k(n) \sin ^{n} \alpha,
$$

where $N_{\mathrm{m}}$ is the maximum electron density in the equatorial plane, $h_{\mathrm{e}}$ is the ratio of the field at a radiation source point (along the line of sight) to the field value along the same field line in the equatorial plane $\left(h_{\mathrm{e}}\right.$ designates the coordinate along the magnetic field line for a given transverse coordinate $L$ ). The parameters $L$ and $h_{\mathrm{e}}$ are functions of the coordinate $r$ along the line of sight: $L=L(r), h_{\mathrm{e}}=h_{\mathrm{e}}(r)$. The constant $k(n)$ is defined by the normalization condition for the pitch angle distribution

$$
\begin{aligned}
& 2 \pi \int_{0}^{\pi} k(n) \sin ^{n+1} \alpha d \alpha=1, \\
& k(n)=\frac{1}{2 \pi \sqrt{\pi}} \Gamma\left(\frac{n+3}{2}\right)\left[\Gamma\left(\frac{n+2}{2}\right)\right]^{-1},
\end{aligned}
$$

where $\Gamma(x)$ is the gamma function.

These relations yield an expression for calculating the emission brightness temperature

$$
T_{\text {eff }}=\frac{2 \pi e^{2}}{\sqrt{3} k_{B}} \frac{c}{v} \frac{k(n)}{\tau K_{2}(1 / \tau)} \int d r N(r)\left(\frac{\sin \mu}{\sqrt{h_{e}}}\right)^{n} I_{\mathrm{M}}\left(\tau, x_{\mathrm{M}}\right),
$$

where the integration is performed along the line of sight and the following notations are introduced: $x_{\mathrm{M}}=v / v_{\mathrm{M}}$,

$$
\begin{aligned}
& v_{\mathrm{M}}=v_{c} / \gamma^{2}=3 e H \sin \mu /(4 \pi m c), \\
& I_{\mathrm{M}}\left(\tau, x_{\mathrm{M}}\right)=\frac{1}{x_{\mathrm{M}}} \int_{1}^{\infty} \gamma \sqrt{\gamma^{2}-1} \exp \left(-\frac{\gamma}{\tau}\right) F\left(\frac{x_{\mathrm{M}}}{\gamma^{2}}\right) d \gamma .
\end{aligned}
$$

In (4), we use the condition that at high harmonics of the gyrofrequency $\left(q=v / v_{\mathrm{He}}>>1, v_{\mathrm{He}}\right.$ is the electron gyrofrequency), the relativistic electron emission is concentrated in a narrow solid angle along the direction of the electron velocity, therefore we can approximately set $\sin \alpha \approx \sin \mu$. In calculations, the function $I_{M}\left(\tau, x_{M}\right)$, as well as $F(x)$, is convenient to use in tabulated form (see Table 1).

For sufficiently large $x_{M}\left(>10^{3}\right)$, we can use the asymptotic approximation of $I_{\mathrm{M}}\left(x_{\mathrm{M}}, \tau\right)$ :

$I_{\mathrm{M}} \approx \sqrt{\frac{2}{3}} \pi \tau \sqrt{1-\left(\frac{3}{2 \tau \xi}\right)^{2}} \exp (-\xi) \approx \sqrt{\frac{2}{3}} \pi \tau \exp (-\xi)$,

where $\xi=(3 / 2 \tau)\left(2 x_{\mathrm{M}} \tau\right)^{1 / 3}>>1$.

To calibrate the calculation method adopted here, we can use simultaneous measurements of synchrotron radio emission intensity [Ochs et al., 1963; Dyce, Horowitz, 1963; Peterson, Hower, 1963) and characteristics of relativistic electron fluxes of the artificial RB during the space nuclear test [Van Allen et al., 1963; Hess, 1963; Peterson, Hower, 1963]. For equatorial stations, emission brightness temperature (4) in the zenith $\left(\mu=\pi / 2, h_{\mathrm{e}}=1\right)$ 


$$
\begin{gathered}
T_{\text {eff }}=\frac{2 \pi e^{2}}{\sqrt{3} k_{\mathrm{B}}} \frac{c}{v} \frac{k(n)}{\tau K_{2}(1 / \tau)} \int R_{\mathrm{E}} d L N(L) I_{\mathrm{M}}\left(\tau, x_{\mathrm{M}}\right) \approx \\
\approx \\
\frac{2 \pi e^{2}}{\sqrt{3} k_{\mathrm{B}}} \frac{c}{v} \frac{k(n)}{\tau K_{2}(1 / \tau)} N_{\mathrm{m}} \sqrt{\pi} R_{\mathrm{E}} \Delta L I_{\mathrm{M}}\left(\tau, x_{\mathrm{M}}^{*}\right),
\end{gathered}
$$

where we approximately estimate integral (4) with Gaussian function (3) for $\Delta L<<L_{\mathrm{m}}, x_{\mathrm{M}}{ }^{*}=\left(2 \mathrm{v} /\left(3 v_{0}\right)\right) L_{\mathrm{m}}{ }^{3}-$ the value of $x_{\mathrm{M}}$ computed at the maximum electron flux with $L=L_{\mathrm{m}}, v_{0}=0.87 \mathrm{MHz}$ is the electron gyrofrequency near Earth's surface at the equator $(L=1)$.

From satellite measurements of electron fluxes in the magnetosphere in July-October 1962 [Van Allen et al., 1963; Hess, 1963] it follows that most electrons of the artificial $\mathrm{RB}$ filled the shells $L \approx 1.2 \div 2$ with maximum electron fluxes at $L_{\mathrm{m}} \approx 1.23 \div 1.6$. In the first $1-2$ hours after the explosion, rapid changes occurred in the configuration of the electron cloud on $L_{\mathrm{m}} \approx 1.23$ with $\Delta L \approx$ 0.13 [Van Allen et al., 1963 ] (Figure 1), followed by slow changes in $L_{\mathrm{m}}, \Delta L, N_{\mathrm{m}}$, and radio emission intensity. The most reliable data on electron fluxes were obtained a few days after the explosion. Let us take the flux value three weeks after the explosion as a reference level $J_{2} \approx 8 \cdot 10^{8} \mathrm{~cm}^{-2} \mathrm{~s}^{-1}$ [Peterson, Hower, 1963]. Using the time factor $\left(1+t_{2} / \tau\right)^{-1}$ [Ochs et al., 1963 ], where $\tau \approx 60$ days and $t_{2}=21$ days, we obtain an estimate of the electron flux for the day of the explosion on July 9, 1962, after the uniform distribution of electrons over drift shells: $J \approx 1.35 J_{2} \approx 1.1 \cdot 10^{9} \mathrm{~cm}^{-2} \mathrm{~s}^{-1}$. Since immediately after the explosion the drift velocity of the electron cloud $d \lambda / d t \sim 15^{\circ}$ $\mathrm{min}^{-1}$ and the duration of the first burst of radio emission (Figure 1) at a level of $50 \%$ from maximum $\Delta t_{0.5} \approx 6 \mathrm{~min}$, the longitude size of the cloud at that instant $\Delta \lambda_{0.5} \approx 90^{\circ}$. After the uniform distribution of the cloud along the drift shells, its size became $\Delta \lambda \approx 360^{\circ}$, and the flux density correspondingly fell no less than four times. Hence, the electron flux intensity during the first burst was at least four times higher and can be estimated as $J_{1} \approx 4.4 \cdot 10^{9} \mathrm{~cm}^{-2} \mathrm{~s}^{-1}$. Further we assume that the electron flux density $J_{\mathrm{m}}=c N_{\mathrm{m}}$, where $c$ is the velocity of light.

From the degree of decrease in the synchrotron radio emission intensity measured at stations at different dis-

tances from the equator [Dyce, Horowitz, 1963], we can estimate the degree of electron anisotropy for $N \propto \sin ^{n} \alpha$ as $n \approx 5 \div 6$. The equilibrium energy spectrum of electrons during fission of $U^{235}$ in the interval $1 \mathrm{MeV}<E<7 \mathrm{MeV}$ ( $E$ is the kinetic energy) has the form $d n / d E \sim \exp \left(-0.575 E-0.055 E^{2}\right) \quad$ [Van Allen et al., 1963 ] and is not Maxwellian. Distribution (2) is best approximated to the $\mathrm{U}^{235}$ fission spectrum at $\tau \approx 1.4 \div 1.5$ $\left(T_{\mathrm{e}}=715 \div 750 \mathrm{keV}\right)$.

Table 2 lists synchrotron radio emission temperatures observed in 1962 [Ochs et al., 1963; Peterson and Hower, 1963] and calculated with (5) ( $\Delta T_{\text {obs }}$ and $\left.\Delta T_{\text {calc }}\right)$ at $50 \mathrm{MHz}$ for several instants after the explosion. Given the roughness of the estimates assuming an accuracy up to a coefficient, we can note good agreement between $\Delta T_{\text {calc }}$ and observations. Figure 3 shows the frequency dependence of brightness temperatures, which also demonstrates agreement between calculated $\Delta T_{\text {eff }}$ and those observed in 1962

\section{SKY-DISTRIBUTION OF INTENSITY OF SYNCHROTRON RADIO EMISSION FROM MAGNETO- SPHERIC RELATIVISTIC ELECTRONS}

For an observation point located at an arbitrary latitude, when calculating integral (4) along a direction, the line-of-sight path is set in a coordinate system with the origin at an observation point on Earth's surface and Zaxis directed to the zenith, whereas the dipole magnetic field vector and its components, as well as the electron density are $N(\vec{R})$, calculated in a coordinate system with the origin at the center of Earth and Z-axis directed to the celestial pole. Coordinates of an emitting volume element must be determined in both the coordinate systems. When calculating from (1)-(4), we use standard transformation formulas for the transition between the coordinate systems, as well as dipole magnetic field formulas.

Table 1

Values of $I_{\mathrm{M}}\left(x_{\mathrm{M}}, \tau\right)$ for different $x_{\mathrm{M}}$ and $\tau$

\begin{tabular}{|c|c|c|c|c|c|c|c|c|c|c|c|c|c|}
\hline$x_{\mathrm{M}}$ & 1 & 2 & 5 & 10 & 20 & 50 & 100 & 200 & 500 & 1000 & 2000 & 5000 & 10000 \\
\hline$\tau=1$ & 1.286 & 0.667 & 0.234 & 0.089 & 0.027 & $3.8 \cdot 10^{-3}$ & $5.8 \cdot 10^{-4}$ & $5.5 \cdot 10^{-5}$ & $9.9 \cdot 10^{-7}$ & $1.9 \cdot 10^{-8}$ & $1.4 \cdot 10^{-10}$ & $2.6 \cdot 10^{-14}$ & $5.6 \cdot 10^{-18}$ \\
\hline$\tau=2$ & 9.601 & 5.462 & 2.370 & 1.145 & 0.494 & 0.128 & 0.036 & $7.8 \cdot 10^{-3}$ & $5.8 \cdot 10^{-4}$ & $4.7 \cdot 10^{-5}$ & $2.0 \cdot 10^{-6}$ & $8.8 \cdot 10^{-9}$ & $4.2 \cdot 10^{-11}$ \\
\hline$\tau=3$ & 27.852 & 16.402 & 7.686 & 4.061 & 1.984 & 0.650 & 0.234 & 0.069 & $9.0 \cdot 10^{-3}$ & $1.3 \cdot 10^{-3}$ & $1.1 \cdot 10^{-4}$ & $1.7 \cdot 10^{-6}$ & $2.9 \cdot 10^{-8}$ \\
\hline$\tau=4$ & 57.618 & 34.530 & 16.827 & 9.311 & 4.852 & 1.798 & 0.739 & 0.258 & 0.046 & $8.9 \cdot 10^{-3}$ & $1.2 \cdot 10^{-3}$ & $3.7 \cdot 10^{-5}$ & $1.2 \cdot 10^{-6}$ \\
\hline
\end{tabular}

Table 2

Experimental $\Delta T_{\text {obs }}$ [Ochs et al., 1963; Peterson, Hower, 1963] and calculated $\Delta T_{\text {calc }}$ brightness temperatures of synchrotron radio emission at $50 \mathrm{MHz}$

\begin{tabular}{|c|c|c|c|c|c|c|c|c|}
\hline time relative to the explosion $(t=0)$ & $I, \mathrm{~mA}$ & $T_{\text {obs }}, K$ & $\Delta T_{\mathrm{obs}}, K$ & $L_{\mathrm{m}}$ & $\Delta L$ & $\tau$ & $J, \mathrm{~cm}^{-2} \mathrm{~s}^{-1}$ & $\Delta T_{\mathrm{calc}}, K$ \\
\hline$t=-1 \mathrm{~min}$ (b a ckground) & 12 & 5742 & - & - & - & - & - & - \\
\hline$t=+6 \mathrm{~m} \mathrm{in}$ & 105 & $5.0 \cdot 10^{4}$ & $4.45 \cdot 10^{4}$ & 1.23 & 0.13 & 1.5 & $4.40 \cdot 10^{9}$ & $3.60 \cdot 10^{4}$ \\
\hline$t=+3 \mathrm{~h} \mathrm{r}$ & 30 & $1.4 \cdot 10^{4}$ & $0.86 \cdot 10^{4}$ & - & - & 1.5 & $1.10 \cdot 10^{9}$ & - \\
\hline$t=+3 \mathrm{w}$ e e k s & - & - & $0.32 \cdot 10^{4}$ & 1.55 & 0.35 & 1.5 & $0.80 \cdot 10^{9}$ & $0.33 \cdot 10^{4}$ \\
\hline
\end{tabular}




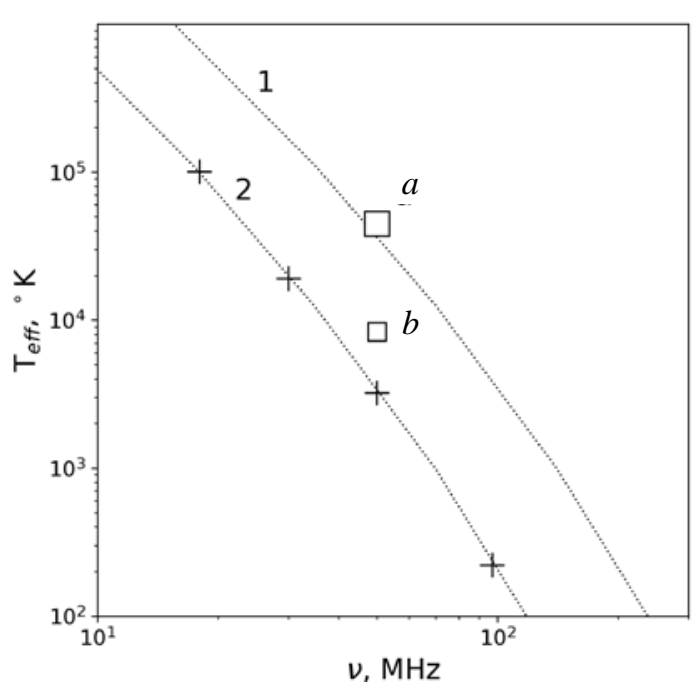

Figure 3. Brightness temperatures of radio emission from the artificial RB, measured at equatorial stations at the zenith in 1962: crosses mark temperatures 3 weeks after the explosion at frequencies of 18, 30, 50, and $100 \mathrm{MHz}$ [Peterson, Hower, 1963], squares indicate radio emission temperatures at a frequency of $50 \mathrm{MHz}$, measured in $6 \min (a)$ and $3 \mathrm{hrs}(b)$ after the explosion (see Figure 1). Dotted lines show calculated curves at $\tau=1.5$ and $n=5$ for two configurations of the artificial RB: $\mathrm{J}_{\mathrm{m}}=4.4 \cdot 10^{9} \mathrm{~cm}^{-2} \mathrm{~s}^{-}$ ${ }^{1}, L_{\mathrm{m}}=1.23, \Delta L=0.13$ (configuration 1 ); $J_{\mathrm{m}}=0.8 \cdot 10^{9} \mathrm{~cm}^{-2} \mathrm{~s}^{-1}, L_{\mathrm{m}}=1.55, \Delta L=0.35$ (configuration 2)

Figure 4 illustrates variations in brightness temperature of synchrotron emission in the zenith and in the direction of the celestial pole when the geomagnetic (dipole) latitude of an observation point $\Phi_{0}$ changes. This Figure also shows the impact of electron flux anisotropy on brightness temperature. It can be seen that beyond the equatorial region $\left(\left|\Phi_{0}\right|>40^{\circ}\right)$ the synchrotron radio emission can actually be observed only with isotropic electron fluxes. From Figure 4 it also follows that for both the directions in an isotropic electron flux $(n=0)$ when the electron density along the flux tube is constant, the maximum brightness temperature is shifted to the equator relative to the center of the $\mathrm{RB}$ base $L_{\mathrm{m}}=1 / \cos ^{2} \Phi_{\mathrm{m}}$ by approximately half-width of RB: $\Delta \Phi \approx \Delta L\left(2 L_{m} \sqrt{L_{m}-1}\right)^{-1}$. On Earth's surface at $\Delta L=0.5$, this corresponds to $\Delta \Phi \approx 1.4^{\circ} \div 3.4^{\circ}(160-375 \mathrm{~km})$ when $L_{\mathrm{m}}$ varies from 5 to 3 . When RB moves to smaller $L$ -

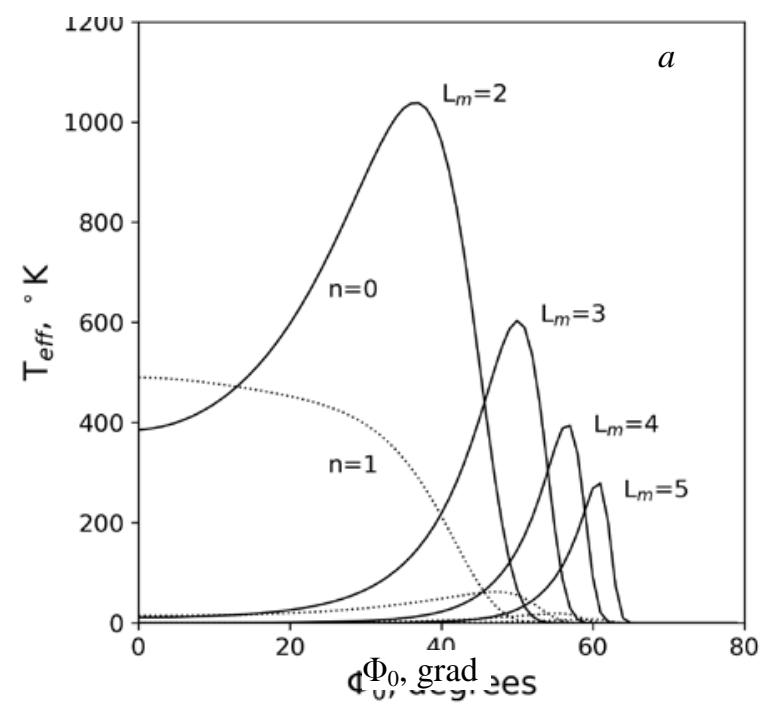

shells with other characteristics $\left(\Delta L, \tau, n, J_{\mathrm{m}}\right)$ being constant, the intensity in the emission maximum increases due to an increase in the angle between emission direction and magnetic field vector.

Figure 5 shows two-dimensional sky-distributions synchrotron radio emission intensity for three RB configurations, approximately 10-100 times less intense than the artificial RB in July 1962. The distributions exhibit two bright regions that are symmetric with respect to the meridian. Their formation is due to the joint action of two factors: a) more favorable aspect angles between magnetic field vector and emission direction; b) greater optical thickness of the region from which emission is recorded. Emission in these directions is several times more intense than in the plane of the celestial meridian, toward which riometer antennas are usually pointed.

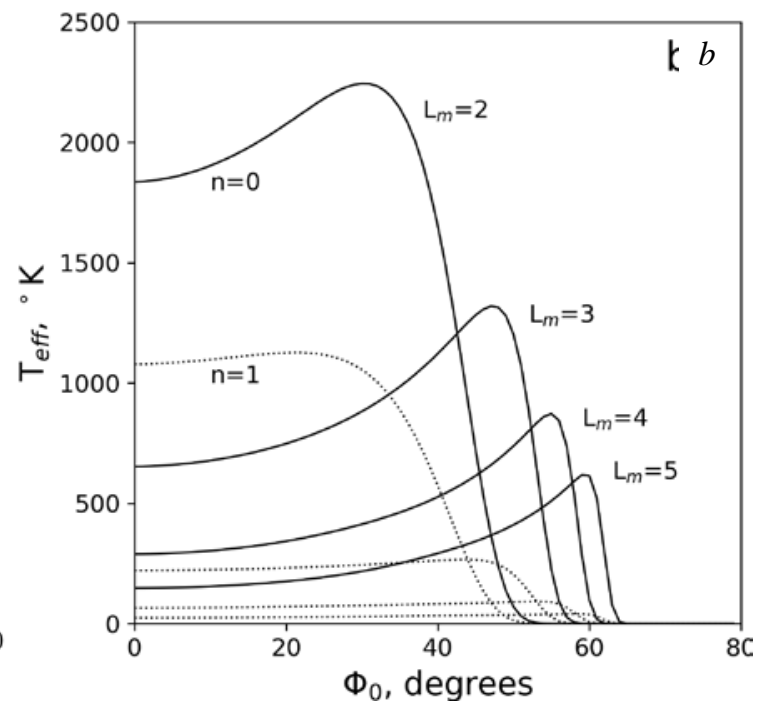

Figure 4. Brightness temperature of emission at a frequency of $30 \mathrm{MHz}$ at the zenith $(a)$ and toward the celestial pole $(b)$ versus a geomagnetic latitude of an observer $\Phi_{0}$ for different RB positions $\left(J_{\mathrm{m}}=3 \cdot 10^{7} \mathrm{~cm}^{-2} \mathrm{~s}^{-1}, \tau=2, \Delta L=0.5\right)$; the solid line is the isotropic distribution over pitch angles $(n=0)$, the dashed line is the anisotropic distribution $(n=1)$ 

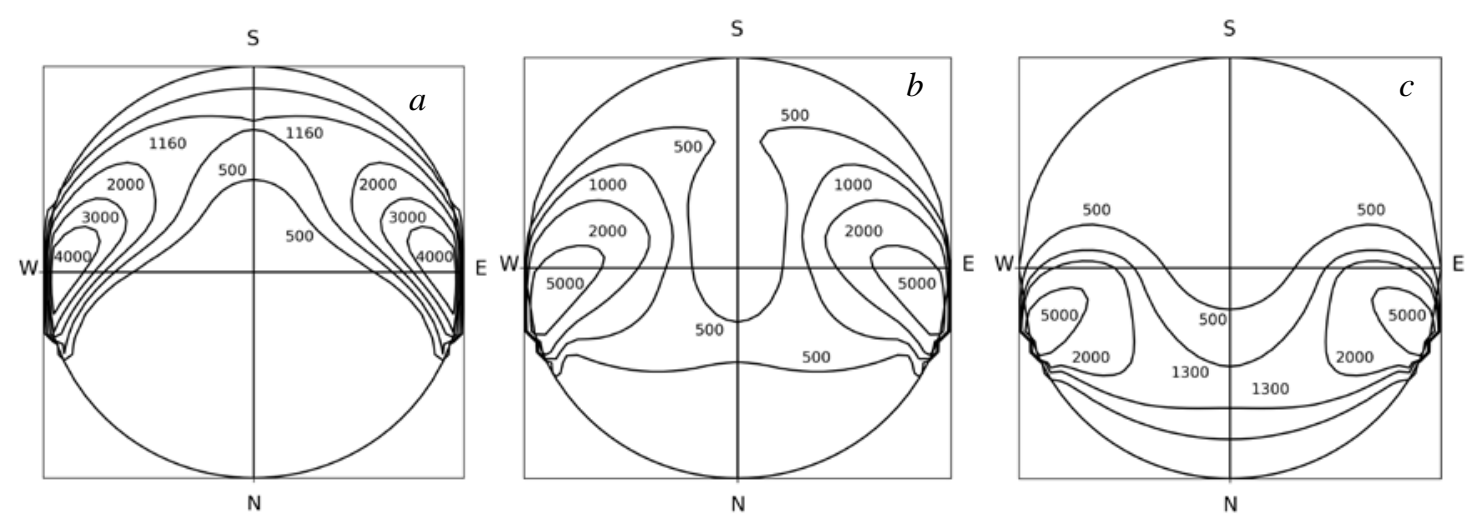

Figure 5. Sky-distribution of brightness temperature $T_{\text {eff }}(\mathrm{K})$ at $v=30 \mathrm{MHz}$ for an observer at $\Phi_{0}=60^{\circ}(L=4)$ with RB characteristics: $L_{\mathrm{m}}=3.5, \tau=1.5\left(T_{\mathrm{e}}=750 \mathrm{keV}\right), J_{\mathrm{m}}=5 \cdot 10^{7} \mathrm{~cm}^{-2} \mathrm{~s}^{-1}(a) ; L_{\mathrm{m}}=4.0, \tau=1.0(500 \mathrm{keV}), J_{\mathrm{m}}=1 \cdot 10^{8} \mathrm{~cm}^{-2} \mathrm{~s}^{-1}(b) ; L_{\mathrm{m}}=4.5, \tau=0.5(250$ $\mathrm{keV}), J_{\mathrm{m}}=3 \cdot 10^{8} \mathrm{~cm}^{-2} \mathrm{~s}^{-1}(c)$; in all cases, $n=0, \Delta L=0.5$. In the center of the images is the zenith, the circle is the horizon

The intensity of emission (or absorption) detected with riometers are usually measured in decibels relative to the undisturbed cosmic noise level $T_{\mathrm{N}}$. Given radio wave absorption in the lower ionosphere, the relative radio noise level is

$$
\begin{aligned}
& S(\mathrm{~dB})=10 \lg \left[\frac{\left(T_{\mathrm{N}}+T_{\mathrm{eff}}\right) e^{-\tau^{\prime}}}{T_{\mathrm{N}}}\right]= \\
& =-\Gamma(\mathrm{dB})+4.34 \ln \left(1+\frac{T_{\mathrm{eff}}}{T_{\mathrm{N}}}\right) \approx-\Gamma(\mathrm{dB})+4.34 \frac{T_{\mathrm{eff}}}{T_{\mathrm{N}}},
\end{aligned}
$$

where $\tau^{\prime}$ is the optical thickness of the $\mathrm{D}$ region, $\Gamma=4.34$ $\tau^{\prime}$ is the corresponding radio wave absorption in the $\mathrm{D}$ region in decibels, $T_{\mathrm{N}}$ is the brightness temperature of the background cosmic radio noise, $T_{\text {eff }}$ is the temperature of the radio noise generated in the magnetosphere or in the ionosphere above the $\mathrm{D}$ region. The latter approximation in (6) refers to a practically significant case $T_{\text {eff }}<<T_{\mathrm{N}}$. Assuming that for riometers the root-meansquare error of the measurement method is $\Delta S_{\mathrm{rms}}=0.1 \mathrm{~dB}$ [Little, Leinbach, 1958; Driatskii 1974], and the cosmic noise temperature outside the galactic plane at a frequency of $30 \mathrm{MHz} T_{\mathrm{N}} \approx 20000 \mathrm{~K}$ [Allen, 1977], we have a minimum change in the emission temperature $\Delta T_{\min }=460 \mathrm{~K}$, which in the absence of absorption $(\Gamma=0)$ can be detected with a riometer at $30 \mathrm{MHz}$.

To get a picture of spatial distribution of relative synchrotron emission intensity $S(\mathrm{~dB})$, we should specify the sky-distribution of cosmic noise intensity $T_{\mathrm{N}}(\chi, A), \chi$ is the zenith angle, $A$ is the azimuth. The distribution of $T_{\mathrm{N}}(\chi, A)$ depends on geographic coordinates of observation point and on sidereal time. To obtain this distribution, a map of isolines of brightness temperature of cosmic radio emission at $200 \mathrm{MHz}$ in the galactic coordinate system is used [Kraus, 1973]: $T_{\mathrm{N}}(b, l)=$ const, $b, l$ are the galactic latitude and longitude. Then, this map through formulas for transforming coordinates from the galactic system to the equatorial one [Astronomical Calendar, 1981] is transformed into a map of isolines $T_{\mathrm{N}}(\alpha, \delta)=$ const, where $\alpha$ is the right ascension, $\delta$ is the declination. Then, for the given geographic coordinates of the observation point and sidereal time at the moment of observations, formulas for transforming equatorial coordinates into horizontal ones [Astronomical Calen- dar, 1981 ] are used to calculate isolines of $T_{\mathrm{N}}(\chi$, A) $=$ const at $200 \mathrm{MHz}$. Using the frequency dependence of the galactic radio emission intensity in the form of $I_{v} \sim(1 / v)^{a}$, where in the frequency range $30-200 \mathrm{MHz}$ the power exponent is approximately equal to $a \approx 0.4 \div 0.5$ [Yetes, Wielebinski, 1967], we obtain the distribution of brightness temperature of cosmic noise at $v$ in the form of $T_{v}(\chi, A)=[200 / v(\mathrm{MHz})]^{2.5} T_{200}(\chi, A)$.

Figure 6, a shows the distribution of brightness temperature of cosmic noise at $50 \mathrm{MHz}$ in the sky over Jicamarca in July 1962. In panel $b$, the picture of cosmic radiation is supplemented with synchrotron emission from the artificial RB; panel $c$ shows the synchrotron emission intensity in decibels against the background emission. It is noteworthy that six minutes after a nuclear explosion, the galactic radio emission is almost completely masked by emission from the artificial RB (Figure 6, $b$, upper panel). If Earth had such RBs constantly, the history of terrestrial radio astronomy would be different from that we have now. On the other hand, simple estimates show that for an off-Earth observer even such a strong environmental impact as a $1.4 \mathrm{Mt}$ nuclear explosion in the magnetosphere and the formation of an artificial RB can be detected from its synchrotron emission ( $v=50 \mathrm{MHz}$ ) only at astronomically short distances: $r(\mathrm{AU})<2.6 \cdot 10^{-5} \mathrm{D}(\mathrm{m})$, where $r(\mathrm{AU})$ is the distance in astronomical units, $1 \mathrm{AU}=150$ millions of $\mathrm{km}, D$ is the antenna size in meters. For example, the corresponding technological activity on Earth can be detected with a VHF antenna $100 \mathrm{~m}$ in diameter no further than from the lunar orbit $\left(2.56 \cdot 10^{-3} \mathrm{AU}\right)$.

\section{FREQUENCY DEPENDENCE OF SYNCHROTRON RADIO EMIS- SION INTENSITY (dB)}

According to (6), the frequency dependence of $S$ (dB) for $T_{\text {eff }}<<T_{\mathrm{N}}$ and $\Gamma=0$ is $S(\mathrm{~dB}) \sim T_{\text {eff }}(v) / T_{\mathrm{N}}(v)$. To construct frequency characteristics of $S(\mathrm{~dB})$ presented in Figure 7, we use the spectral dependence of the background cosmic noise: $T_{\mathrm{N}}(v)=2 \cdot 10^{4}(30 / v)^{2.5}$, where $T_{\mathrm{N}}$ is in $\mathrm{K}, v$ is in $\mathrm{MHz}$ [Allen, 1977; Yetes, Wielebinski, 1967]. With increasing electron energy, the relative contribution of high harmonics to the total energy of its synchrotron emission increases. Against a decrease in 
$T_{\mathrm{N}}(v)$ at high frequencies, this leads to the formation of maximum $S_{\max }(\mathrm{dB})$ at $v_{\max }$. With increasing characteristic electron energy, $S_{\max }$ increases and the position of the maximum shifts to higher frequencies approximately according to the law $v_{\max } \sim \tau^{2} \sim T_{\mathrm{e}}^{2}$. Figure 7 shows that at $T_{\mathrm{e}}=1 \mathrm{MeV}$, the maximum $S(\mathrm{~dB})$ is about $50-60 \mathrm{MHz}$, and at $T_{\mathrm{e}}=2 \mathrm{MeV}$, it is about 200-250 MHz. We can assume that the radio emission events recorded during magnetospheric disturbances at $80 \mathrm{MHz}$ [Chivers, Wells, 1959] and $225 \mathrm{MHz}$ [Eriksen, Harang, 1969] are attributed to the synchrotron emission of electrons with such high characteristic energies. If an observation point is located close to the RB base, we should take into account radio wave absorption in the $\mathrm{D}$ region, caused by atmosphere ionization by precipitating energetic electrons. The amount of absorption is inversely proportional to the square of frequency: $\Gamma(v) \sim v^{-2}$ [Driatskii, 1974]. In the frequency range, where $\Gamma(v) \geq 4.34 \mathrm{~T}_{\text {eff }}(v) / T_{\mathrm{N}}(v)$, the synchrotron radio emission of magnetospheric electrons is undetectable. In Figure 7, dashed curves indicate the frequency dependence of $S(\mathrm{~dB})$ at $\Gamma(30$ $\mathrm{MHz})=0.5 \mathrm{~dB}$, which suggests that the detection condition for the synchrotron emission is realized only above a certain minimum frequency $v \geq v_{\min }$. Referring to Figure 7, the threshold condition $S>\Delta S_{\text {min }} \approx 0.1 \mathrm{~dB}$ with the given absorption holds for $v>v_{\min } \approx 60 \div 70 \mathrm{MHz}$.

\section{DISCUSSION}

Calculations show that the synchrotron emission level to exceed the background cosmic noise level by
$\Delta S_{\text {min }} \approx 0.1 \mathrm{~dB}$ needs rather intense isotropic fluxes of relativistic electrons. The satellite S3-3 for 14 months of observations has recorded 313 cases of precipitation of relativistic electrons with isotropic fluxes from the upper hemisphere [Thorne, Andreoli, 1983]. Isotropic electron fluxes are driven by strong diffusion along the pitch angle during interaction of energetic electrons with waves in the magnetosphere. According to the nature of the angular and energy spectra of precipitating electrons and ions, Thorne and Andreoli [1983] have identified 7 of 313 cases as electron scattering by whistler waves (dawnside), 4 cases as scattering by electromagnetic ion-cyclotron waves (Pc1, duskside), and 302 cases as scattering by electrostatic ion-cyclotron waves (nightside, latitudes characteristic of the auroral oval). Owens and Frank [1968] present isolines of electron fluxes in time- $L$-shell coordinates for two months of the Explorer-14 operation with time averaging over about 1 day. During these two months, the time-smoothed electron fluxes in disturbed periods increased to $J_{\mathrm{m}}(E>230$ $\mathrm{keV}) \geq 10^{7} \mathrm{~cm}^{-2} \mathrm{~s}^{-1}$ and $J_{\mathrm{m}}(E>1.6 \mathrm{MeV}) \geq 10^{6} \mathrm{~cm}^{-2} \mathrm{~s}^{-1}$ on $4 \leq L \leq 6$. It is likely that during very high magnetospheric activity against the smoothed values there were shortterm (about $1 \mathrm{hr}$ ) several-fold enhancements of fluxes as compared to the smoothed ones. For example, from [Vernov et al., 1972; Savenko et al., 1979], which analyzed satellite data on relativistic electron fluxes for 1958-1971, it follows that flux intensities can vary by 2-3 orders of magnitude depending on specific geomagnetic conditions.
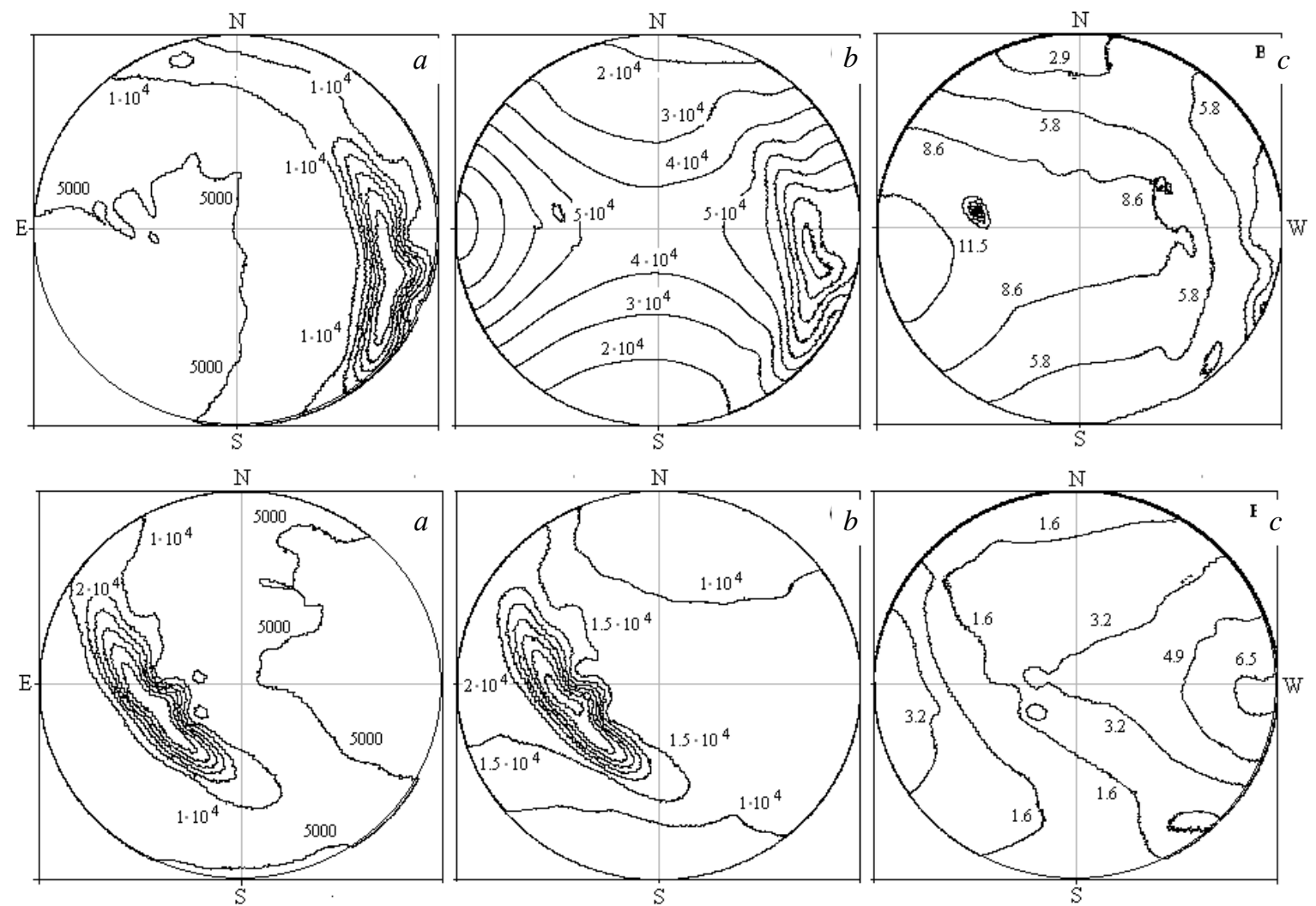

Figure 6. Isolines of brightness temperatures at $50 \mathrm{MHz}$ in the sky over Jicamarca in July 1962: $a$ is the galactic radio emission in $\mathrm{K}, b$ is the sum of cosmic radio emission and synchrotron radio emission from magnetospheric electrons in $\mathrm{K}, c$ is the excess of synchrotron emission over the cosmic noise background in $\mathrm{dB}$; the top panel, 6 min after the explosion; the bottom panel, 3 weeks after the explosion (at $00 \mathrm{UT}$ ) 


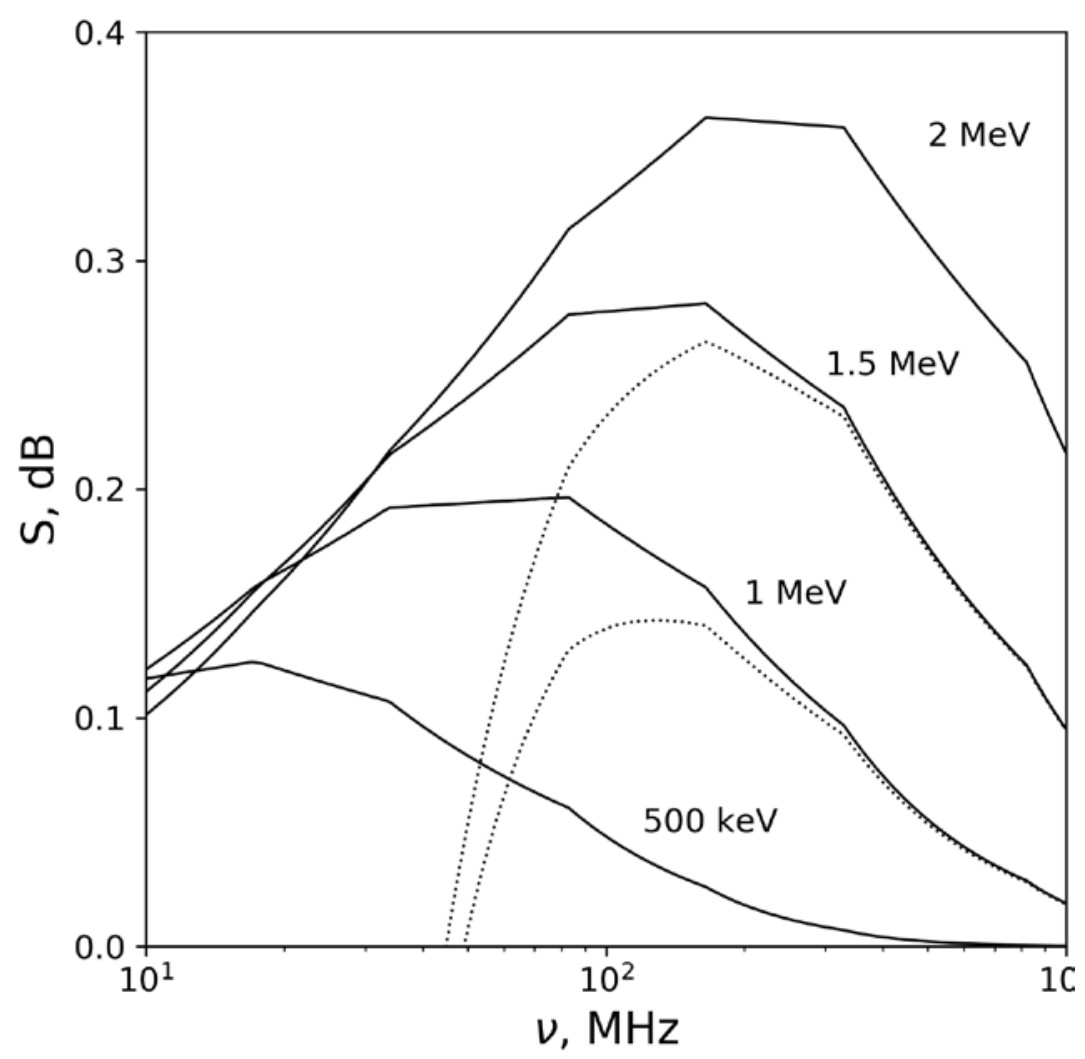

Figure 7. Frequency dependence of relative synchrotron emission intensity $S(\mathrm{~dB})$ for various $T_{\mathrm{e}}\left(J_{\mathrm{m}}=3 \cdot 10^{7} \mathrm{~cm}^{-2} \mathrm{~s}^{-1}, n=0\right.$, $\left.L_{\mathrm{m}}=4.5, \Delta L=0.5, \Phi_{0}=62^{\circ}\right)$ toward the celestial pole. Dashed curves show the effect of absorption $\Gamma(30 \mathrm{MHz})=0.5 \mathrm{~dB}$ with a frequency dependence $\Gamma \sim 1 / v^{2}$ on $S(\mathrm{~dB})$ at $T_{\mathrm{e}}=1 \mathrm{MeV}$ and $T_{\mathrm{e}}=1.5 \mathrm{MeV}$.

For electrons with $E>500 \mathrm{keV}$ there were cases in which maximum fluxes in the outer RB exceeded $J_{\mathrm{m}} \geq 10^{8} \mathrm{~cm}^{-2} \mathrm{~s}^{-1}$ [Vernov et al., 1972]. Electron fluxes with $E>1 \mathrm{MeV}$ exhibited maximum values of $J_{\mathrm{m}} \geq 10^{7}$ $\mathrm{cm}^{-2} \mathrm{~s}^{-1}$ [Savenko et al., 1979]. According to [Borovsky et al., 2016], in the geostationary orbit region $(L \approx 6.6)$ the electron density with characteristic energies $T_{\mathrm{e}}=150 \div 500 \mathrm{keV}$ during magnetospheric storms reaches $N_{\mathrm{m}} \geq 0.001 \mathrm{~cm}^{-3}$ or $J_{\mathrm{m}} \approx c N_{\mathrm{m}} \geq 3 \cdot 10^{7} \mathrm{~cm}^{-2} \mathrm{~s}^{-1}$. As is shown above (Figures 4, 5), for such fluxes at $30 \mathrm{MHz}$, $\Delta T_{\text {eff }}>500 \mathrm{~K}$ in the meridian plane and higher values (more than $2000 \mathrm{~K}$ ) outside the meridian plane at zenith distances $\chi \geq 45^{\circ}$ are to be recorded, i.e., higher than the sensitivity threshold equal to $\Delta T_{\text {eff }} \approx 460 \mathrm{~K}$ for a $30 \mathrm{MHz}$ riometer.

Assuming that the satellite S3-3 has recorded 313 cases of precipitation of isotropic relativistic electron fluxes for 14 months (426 days), we obtain the probability of one event on average about 0.7 of event per day. The probability that in the precipitation the intensity of the electron flux with energies $E \sim 0.5 \div 1 \mathrm{MeV}$ exceeds $10^{7}-10^{8} \mathrm{~cm}^{-2} \mathrm{~s}^{-1}$ is difficult to estimate. On the one hand, for many years there have been made only a small number of reports on magnetospheric HF radio emission. This means that precipitation of relativistic electrons, presumably capable of generating intense synchrotron radio emission, is a rather rare event. On the other hand, attempts to register synchrotron emission from the magnetosphere in the range 30-200 MHz were episodic, and there is no information about any long-term targeted studies of this phenomenon.
Unlike Figure 5, which shows the distribution of absolute values of brightness temperature for a given latitude of the observation point and various RB characteristics, Figure 8 illustrates radio brightness distributions in decibels versus cosmic radio noise in the sky over three subauroral stations for a given configuration of RB ( $L_{\mathrm{m}}=4.5, \Delta L=0.5$ ). The stations are located on the same meridian (the meridian of the ISTP SB RAS Norilsk Magnetic-Ionospheric Station) and correspond to $L$ shells $L \approx 3.4,4.4,5.4$. Figure 8 indicates that the stations located to the north and south of the RB base at distances $\Delta L \approx 1$ observed almost identical regions of enhanced emission brightness $(0.1-0.2 \mathrm{~dB})$, respectively in the southern and northern celestial hemispheres. In this case, this symmetry results from the large inclination of the magnetic field with almost vertical field lines. At the station located almost under the RB base, the isolines $S \geq 0.2 \mathrm{~dB}$ pass outside the meridian plane at zenith angles $\chi>40^{\circ}$ in the east and west of the station. It is obvious that in these directions the probability of detecting synchrotron emission from magnetospheric electrons is maximum. Noteworthy is the minimum probability of detecting magnetospheric emission toward the galactic center (Figure 8, c, top panel), which makes a diurnal motion over the celestial sphere.

It is useful to briefly consider some general conditions for organizing purposeful observations of synchrotron radio emission of magnetospheric electrons, which increase the probability of a positive result.

Latitude of an observation point. Intense precipitation of relativistic electrons most often occurs in the 
region $L=4 \div 6$ [Thorne, Andreoli, 1983; Owens, Frank, 1968; Friedel, 2002], which corresponds to geomagnetic latitudes $\Phi_{0}=60 \div 66^{\circ}$. Because of the strong influence of absorption in the $\mathrm{D}$ region under the $\mathrm{RB}$ base (see Figure 7), receivers should be placed from the equatorial side of it, at distances $200-400 \mathrm{~km}$, i.e. at geomagnetic latitudes $58-62^{\circ}$, where the probability of the absorption effect is low, whereas the emission intensity is maximum (see Figure 4).

Antenna directivity. The sky-distribution of synchrotron emission intensity is highly inhomogeneous, therefore standard riometer antennas with wide directional patterns are less effective than special, more directional antennas. Generally there are two synchrotron emission intensity maxima in the sky, to the west and east of the meridian at zenith angles $\chi \geq 40^{\circ}$. To increase the probability of detecting synchrotron emission, a receiver antenna should have eastward and westward directional lobes at zenith angles of $40^{\circ}<\chi<\arccos \left(f_{\mathrm{o}} \mathrm{F} 2 / v\right)$, where $f_{\mathrm{o}} \mathrm{F} 2$ is the critical frequency of the ionosphere and $v>f_{\mathrm{o}} \mathrm{F} 2$. The most effective variant of antenna array appears to be a phased array with a multilobed beam and reception of radio emission from each lobe separately, for example, as done in [Detrick, Rosenberg, 1990].

Operating frequencies. Referring to Figure 7 , at $v \leq$ $30 \mathrm{MHz}$, riometers are more sensitive to synchrotron emission of moderately relativistic electrons (less than $0.5 \mathrm{MeV}$ ), but at the same time they are sensitive to absorption that affects emission detection. At frequencies $v>50 \mathrm{MHz}$, riometers are less sensitive to absorption and more sensitive to synchrotron emission of highenergy electrons (greater than $1 \mathrm{MeV}$ ). The most informative observations should be made at two frequencies, for example, in the frequency windows 37.7538.25 $\mathrm{MHz}$ and 79.75-80.25 MHz meant for radioastronomy [Kraus, 1973].
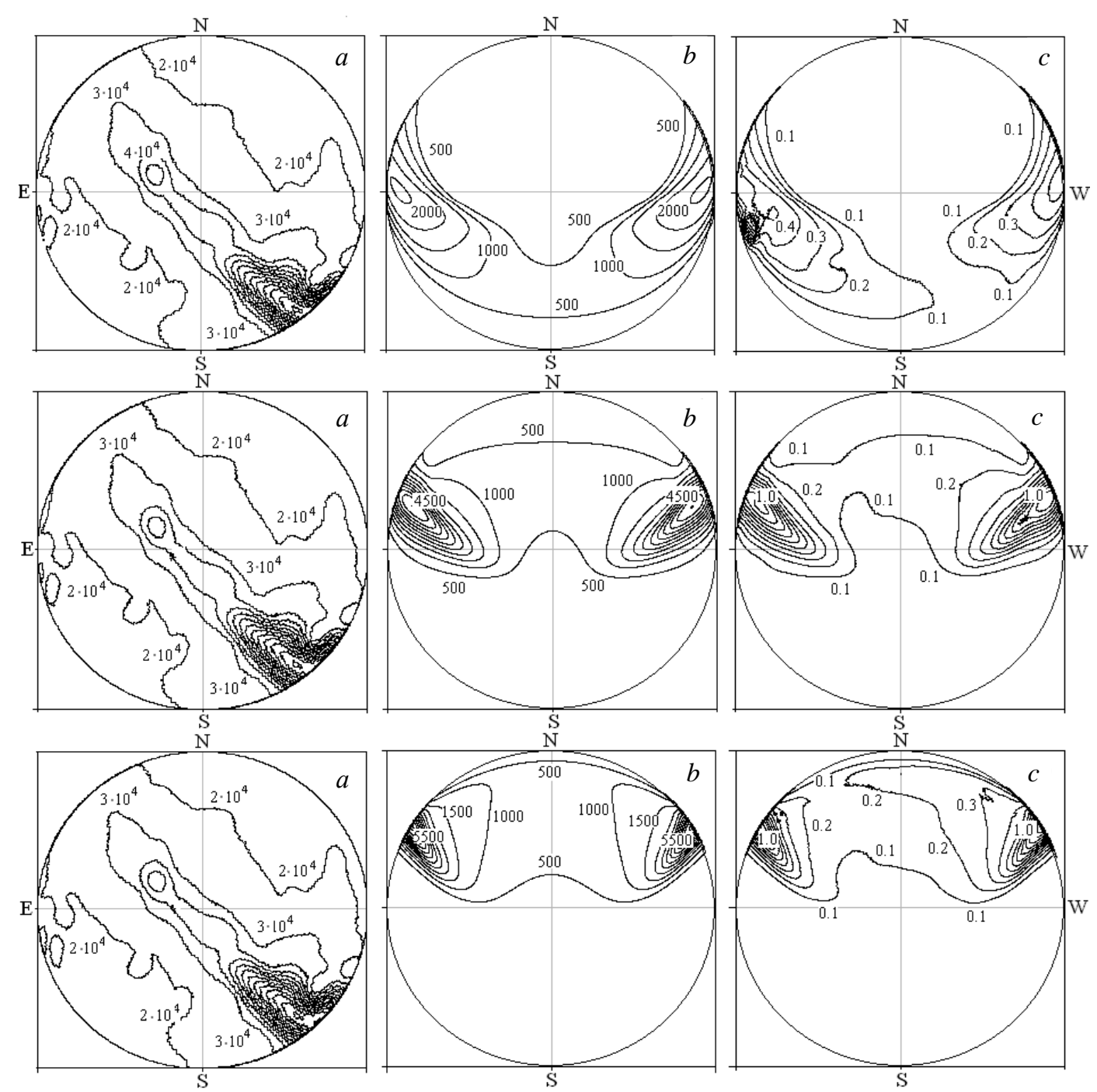

Figure 8. Brightness temperature isolines at $30 \mathrm{MHz}$ in the sky over Norilsk $\left(\Phi_{0}=64.5^{\circ}, L=5.4\right.$, top panel), Turukhansk ( $\Phi_{0}=61.4^{\circ}, L=4.4$, middle panel), and Podkamennaya Tunguska $\left(\Phi_{0}=57.3^{\circ}, L=3.4\right.$, bottom panel) during appearance of RB with $\tau=$ $2\left(T_{\mathrm{e}}=1 \mathrm{MeV}\right), n=0, L_{\mathrm{m}}=4.5, \Delta L=0.5, J_{\mathrm{m}}=3 \cdot 10^{7} \mathrm{~cm}^{-2} \mathrm{~s}^{-1}$ : galactic radio emission in $\mathrm{K}(a)$; synchrotron radio emission of magnetospheric electrons in $\mathrm{K}(b)$, synchrotron emission exceeding the cosmic noise background in decibels $(\Gamma=0 \mathrm{~dB})(c)$. Distributions of the intensity of cosmic radio emission (a) and synchrotron emission in decibels $(c)$ are plotted for sidereal time at 15 UT (21 LT) on September 23, 2017 


\section{CONCLUSION}

The intensity of synchrotron radio emission from magnetospheric electrons is calculated using an RB model with a Gaussian spatial distribution of electron density over $L$-shells and Maxwell's relativistic energy distribution. The results of calculations of emission intensity with this model are consistent with observations of synchrotron emission from the artificial RB in the Starfish nuclear test carried out in 1962; therefore such a model can be employed to calculate the intensity of synchrotron emission from Earth's natural RBs. We have obtained two-dimensional sky-distributions of brightness temperature $T_{\text {eff }}$ of synchrotron radio emission of the model RB and the relative intensity in decibels relative to the level of the galactic radio noise.

The emission intensity in the celestial meridian region, to which riometer antennas are usually pointed, to exceed the detection threshold of $0.1 \mathrm{~dB}$ needs isotropic electron fluxes with a characteristic energy of about $1 \mathrm{MeV}$ and a density of more than $10^{7} \mathrm{~cm}^{-2} \mathrm{~s}^{-}$ ${ }^{1}$. In sky-distributions of synchrotron emission intensity, two symmetrical maxima are formed in the west and east of the meridian at zenith angles greater than $40^{\circ}$. These maxima are an order of magnitude brighter than the celestial meridian. To increase the probability of detecting synchrotron radio emission from magnetospheric electrons, it is preferable to place a receiver at geomagnetic latitudes $58-62^{\circ}$ and use antennas capable of receiving radio emission from various directions. Characteristics of the observed natural magnetospheric HF radio noise such as directions of arrival [Chivers, Wells, 1959; Egan, Peterson, 1960; Ellyett, 1969] and frequency range [Chivers, Wells, 1959; Eriksen and Harang, 1969] agree in principle with the results of calculations of the synchrotron emission from magnetospheric electrons and likely depend on the position of the RB base relative to a receiver and values of relativistic electron temperature.

The work was carried out under the state task of the IAP RAS (project No. 0035-2014-0034).

\section{REFERENCES}

Allen C.W. Astrofizicheskie velichiny [Astrophysical quantities]. Moscow: Mir Publ., 1977. 448 p. (In Russian). English edition: Allen C.W. Astrophysical quantities. University of London. The Athlon Press. 1973. 448 p.

Astronomicheskii kalendar. Postoyannaya chast. [Astronomical Calendar. Basic Part]. Moscow: Nauka Publ., 1981. 704 p. (In Russian)

Borovsky J.E., Cayton T.E., Denton M.H., Belian R.D., Christensen R.A., Ingraham J.C. The proton and electron radiation belts at geosynchronous orbit: Statistics and behavior during high-speed stream-driven storms. J. Geophys. Res.: Space Phys. 2016, vol. 121, pp. 5449-5488.

Chivers H.J.A., Wells H.W. Observations of unusual radiofrequency noise emission and absorption at $80 \mathrm{Mc} / \mathrm{s}$. J. Atmosph. Terr. Phys. 1959, vol. 17, pp. 13-19.

Detrick D.L., Rosenberg T.J. A phased-array radiowave imager for studies of cosmic noise absorption. Radio Sci. 1990, vol. 25, no. 4, pp. 325-338.
Driatskii V.M. Priroda anomalnogo pogloshcheniya kosmicheskogo radioizlucheniya $v$ nizhnei ionosfere vysokikh shirot [The Nature of Anomalous Cosmic Radio Noise Absorption in the Lower Ionosphere of High Latitudes]. Leningrad: Gydrometeoizdat Publ., 1974. 224 p. (In Russian).

Dyce R.B., Horowitz S. Measurements of synchrotron radiation at central Pacific sites. J. Geophys. Res. 1963, vol. 68, no. 3, pp. 713-721. DOI: 10.1029/JZ068i003p00713.

Dyce R.B., Nakada M.R. On the possibility of detecting synchrotron radiation from electrons in the Van Allen belts. $J$. Geophys. Res. 1959, vol. 64, no. 9, pp. 1163-1168.

Egan R.D., Peterson A.M. Auroral noise at HF. J. Geophys. Res. 1960, vol. 65, no. 11, p. 3830.

Ellyett C.D. Radio noise of auroral origin. J. Atmosph. Terr. Phys. 1969, vol. 31, pp. 671-682.

Friedel R.H.W., Reeves G.D., Obara T. Relativistic electron dynamics in the inner magnetosphere - A review. J. Atmos. Solar-Terr. Phys. 2002, vol. 64, no. 1, pp. 57-72.

Eriksen G., Harang L. Radio noise from the ionosphere on $225 \mathrm{MHz}$ during a great ionosphere disturbance. Phys. Norveg. 1969, vol. 4, no. 1, pp. 1-4.

Ginzburg V.L., Syrovatsky S.I. Cosmic synchrotron radiation. Uspehi fizicheskikh nauk [Physics-Uspekhi (Adv. Phys. Sci.)] 1965, vol. 87, no. 1, pp. 65-111. (In Russian).

Hess W.N. The artificial radiation belt made on July 9, 1962. J. Geophys. Res. 1963, vol. 68, no. 3, pp. 667-683. DOI: 10.1029/JZ068i003p00667.

Korchak A.A. On the synchrotron radiation of charged particles in the dipole magnetic field. I. Astronomicheskii Zhurnal [Astronomy Reports]. 1963, vol. 40, pp. 994-1006. (In Russian)

Kraus J.D. Radioastronomiya [Radio Astronomy]. Moscow: Sovetskoe Radio Publ., 1973. 456 p. (In Russian). English edition: Kraus J.D. Radio Astronomy. McGraw-Hill Book Company. New York, St. Louis, San Francisco, Toronto, London, Sydney, 1966.

Little C.G., Leinbach H. Some measurements of highlatitude ionospheric absorption using extraterrestrial radio waves. Proc. IRE. 1958, vol. 46, no. 1, pp. 334-348.

Ochs G.R., Farley Jr. D.T., Bowles K.L., Bandyopadhay P. Observations of synchrotron radio noise at the magnetic equator following the high-altitude nuclear explosion of July 9, 1962. J. Geophys. Res. 1963, vol. 68, no. 3, pp. 701-711. DOI: 10.1029/JZ068i003p00701.

Owens H.D., Frank L.A. Electron omnidirectional intensity contours in the Earth`s outer radiation zone at the magnetic equator. J. Geophys. Res. 1968, vol. 73, no. 1, pp. 199-208. DOI: 10.1029/JA073i001p00199.

Pakholchik A.G. Radioastrofizika [Radio Astrophysics]. Moscow: Mir Publ., 1973. 252 p. (In Russian). English edition: Pacholczyk A.G. Radio Astrophysics. W.H. Freeman \& Company. San Fracisco, 1970.

Peterson A.M., Hower G.L. Synchrotron radiation from high-energy electrons. J. Geophys. Res. 1963, vol. 68, no. 3, pp. 723-734. DOI: 10.1029/JZ068i003p00713.

Potapov A.S. Relativistic electrons of the external radiation belt and methods of their forecast (review). Solar-Terr. Phys. 2017, vol. 3, no. 1, pp. 57-72.

Romanova N.V. et al. Statistical correlation of the rate of failures on synchronous satellites with fluxes of energ ic electrons and protons. Cosmic Res. 2005, vol. 43, no. pp. 179-185.

Savenko I.A., Senchuro I.N., Shavrin P.I. On maximal electron fluxes with $E>1 \mathrm{MeV}$ energy in the Earth's outer radiation belt on 1958-1971. Kosmicheskiye issledovaniya [Cosmic Res.]. 1979, vol. 17, no. 1, pp. 141-145. (In Russian). Thorne R.M., Andreoli L.J. Polyarnaya verkhnyaya at- 
mosfera [Polar Upper Atmosphere]. Moscow: Mir Publ., 1983, pp. 367-379. (In Russian). English edition: Thorne R.M., Andreoli L.J. Mechanism of the relativistic electron intensive precipitation. Exploration of the Polar Upper Atmosphere. Proc. of the NATO Adv. Study Inst. held at Lillehammer, Norway, May 5-16, 1980. Eds. C.S. Deer, J.A. Holtet. D. Reidel. Publ. Comp. Dordrecht - Boston - London, 1980.

Trubnikov B.A. Radiation of plasma in magnetic field. Doklady Akademii nauk [Doklady Physics]. 1958, vol. 118, no. 5, pp. 913-916. (In Russian).

Van Allen J.A., Frank L.A., O’Brien B.J. Satellite observations of the artificial radiation belt of July 1962. J. Geophys. Res. 1963, vol. 68, no. 3, pp. 619-627. DOI: 10.1029/ JZ068i003p00619.

Vernov S.N., Gorchakov E.V., Kuznetsov S.N. et al. The particle fluxes in the outer magnetic field. Fizika magnitosfery [Magnetospheric Phys.]. Eds. D.J. Williams, G.D. Mead. Moscow: Mir Publ., 1972, pp. 318-344. (In Russian).
Vesecky J.F., Peterson A.M. Radio frequency synchrotron radiation from the Van Allen belts. J. Geophys. Res. 1967, vol. 72, no. 5, pp. 1647-1650.

Yetes K.W., Wielebinski R. Observational results of radio sky spectrum. Astrophys. J. 1967, vol. 149, pp. 439-440.

How to cite this article

Klimenko V.V. Sky-distribution of intensity of synchrotron radio emission of relativistic electrons trapped in Earth's magnetic field. Solar-Terrestrial Physics. 2017. Vol. 3, No. 4, P. 32-43. DOI: $10.12737 /$ stp-34201704 